\title{
Milrinone effects on cardiac mitochondria, hemodynamics, and death in catecholamine-infused rats
}

\author{
I-Chun Lin ${ }^{1 凶}$, Chih-Wei Wu' ${ }^{2}$, Ying-Jui Lin ${ }^{1}$, Mao-Hung Lo ${ }^{1}$, Kai-Sheng Hsieh ${ }^{3}$, Julie Y. H. Chan ${ }^{2}$ and Kay L. H. Wu ${ }^{2,4}$ \\ (c) The Author(s), under exclusive licence to the International Pediatric Research Foundation, Inc 2022
}

BACKGROUND: Catecholamine-storm is considered the major cause of enterovirus 71-associated cardiopulmonary death. To elucidate the effect of milrinone on cardiac mitochondria and death, a rat model of catecholamine-induced heart failure was investigated.

METHODS: Young male Spray-Dawley rats received a continuous intravenous infusion of norepinephrine then followed by cotreatment with and without milrinone or esmolol. Vital signs were monitored and echocardiography was performed at indicated time points. At the end of experiments, hearts were extracted to study mitochondrial function, biogenesis, and DNA copy numbers. RESULTS: Hypernorepinephrinemia induced persistent tachycardia, hypertension, and high mortality and significantly impaired the activities of the electron transport chain and suppressed mitochondrial DNA copy number, mitochondrial transcription factor A and peroxisome proliferator-activated receptor-gamma coactivator 1-a. Norepinephrine-induced hypertension could be significantly suppressed by milrinone and esmolol. Milrinone improved but esmolol deteriorated the survival rate. The left ventricle was significantly enlarged shortly after norepinephrine infusion but later gradually reduced in size by milrinone. The impairment and suppression of mitochondrial function could be significantly reversed by milrinone but not by esmolol.

CONCLUSIONS: Milrinone may protect the heart via maintaining mitochondrial function from hypernorepinephrinemia. This study warrants the importance of milrinone and the preservation of mitochondrial function in the treatment of catecholamineinduced death.

Pediatric Research (2022) 92:1309-1315; https://doi.org/10.1038/s41390-022-01964-6

\section{IMPACT:}

- Milrinone may protect the heart from hypernorepinephrinemia-induced death via maintaining myocardial mitochondrial activity, function, and copy number.

- Maintenance of cardiac mitochondrial function may be a potential therapeutic strategy in such catecholamine-induced heart failure.

\section{INTRODUCTION}

Acute fulminant heart failure (HF) is a critical complication of enterovirus 71 (EV-71) infection-related brainstem encephalitis, pheochromocytoma, and pandemic coronavirus infection disease2019 (COVID-19). ${ }^{1-4}$ Severe acute respiratory syndrome coronavirus-2 can induce myocarditis via direct invasion of the myocardium and consequent HF. Of note, viral entry-induced angiotensin-converting enzyme 2 downregulation worsens HF via elevated level of angiotensin II and activation of the renin-angiotensin system, which was especially observed in those accompanied with hypercytokinemia. ${ }^{4}$ Catecholamine-storm, a status of hypernorepinephrinemia resulting from excessive release of catecholamines in these circumstances may cause hypersympathetic activity including tachycardia and hypertension, consequently leading to myocardial damage, ${ }^{5}$ acute fulminant $\mathrm{HF}^{6}$ and death. ${ }^{7,8}$ Without adequate therapy, most of these involved patients would suddenly die within
4-6 $\mathrm{h}$ after the clinical presentation of hypernorepinephrinemia in EV-71-complicated myocarditis. ${ }^{1}$ The mechanism of disease progression and the most effective therapeutic strategy remain uncertain. Despite high mortality in the acute phase, the survivors may develop long-term left ventricular dysfunction and experience poor life quality.

Many studies have suggested that norepinephrine (NE), released due to infection of the vasomotor center, is able to induce apoptosis in neonatal rat cardiomyocytes and endothelial cells through a $\beta$-adrenergic receptor-reactive oxygen species-TNF-caspase signaling pathway. $^{9-11}$ Similar to the cardiac histopathology in patients with NE-associated damage, EV-71-complicated myocarditis revealed coagulative myocytolysis, myofibrillar degeneration, and apoptosis of cardiomyocytes, which are the characteristics of catecholamine cardiotoxicity. ${ }^{1}$ The underlying mechanism is generally believed to result from excessive NE release caused by a brain lesion with

\footnotetext{
${ }^{1}$ Department of Pediatrics, Kaohsiung Chang Gung Memorial Hospital and Chang Gung University College of Medicine, Kaohsiung, Taiwan. ${ }^{2}$ Institute for Translational Research in Biomedicine, Kaohsiung Chang Gung Memorial Hospital, Kaohsiung, Taiwan. ${ }^{3}$ Department of Pediatrics, Shuang Ho Hospital-Taipei Medical University, New Taipei City, Taiwan. ${ }^{4}$ Department of Senior Citizen Services, National Tainan Institute of Nursing, Tainan, Taiwan. ${ }^{凶}$ email: mslinichun@gmail.com; wlh0701@gmail.com
} 
cardiomyocyte apoptosis. ${ }^{9}$ Thus, $\beta$-blockers have been proposed to possibly relieve substantial symptoms and prevent further adverse cardiac dysfunction by reducing myocardium energy requirement. ${ }^{12}$

Mitochondria act as the most metabolically active organelle to support the cardiac energy, ${ }^{11}$ and to generate energy via electron transport chain (ETC) from mitochondrial respiratory complex I and II to III, which then transfers the electron to IV. Catecholamine could depress mitochondrial function and subsequent HF. ${ }^{13,14}$ The progression of HF has been linked to the faltering energy supply, ${ }^{15}$ and suppression of ETC activities. ${ }^{16,17}$ Improvement of ETC efficiency appears to reduce mortality in HF patients. ${ }^{18-20}$ Mitochondrial biogenesis is strictly regulated to maintain the mitochondrial mass and function. Peroxisome proliferatoractivated receptor-gamma coactivator $(P G C)-1 a$ increases the expression of mitochondrial transcription factor A (TFAM) for mitochondrial DNA (mtDNA) replication and mitochondrial genes encoding to generate new mitochondria. ${ }^{21}$ Thus, impairment of mitochondrial biogenesis contributes to the decrease of mitochondrial mass and sequel mitochondrial dysfunction.

In this study, using a rodent model of hypernorepinephrinemia to mimic the fulminant course of HF in children with enterovirus71 infection, we examined whether milrinone or esmolol could effectively rescue catecholamine-induced mortality, hemodynamic alteration, and mitochondrial ETC activities and biogenesis in the myocardium of left ventricle (LV).

\section{METHODS \\ Study design}

Male Spray-Dawley rats (6-week-old) of similar body weight were used (Supplemental Fig. 1). In the beginning, rats were anesthetized with sodium pentobarbital ( $50 \mathrm{mg} / \mathrm{kg}$; intraperitoneally) after isoflurane (3-5\%) induction. Femoral veins were meticulously cannulated, and rats were kept hemodynamically stable at least $30 \mathrm{~min}$ after good sedation with intravenously (i.v.) continuous infusion of pentobarbital. To mimic a rapid and fulminant course of excessive catecholamine-induced HF, NE bitartrate was i.v. infused (NE, $8 \mu \mathrm{g} / \mathrm{kg} / \mathrm{min}$; infusion rate, $4 \mu \mathrm{l} / \mathrm{min}$; China Chemical \& Pharmaceutical Co., Taiwan) via the femoral vein. Normal saline (sal, i.v. infusion rate, $4 \mu \mathrm{l} / \mathrm{min}$ ) was used as a negative control. The treatment groups were saline (i.v. infusion rate, $4 \mu \mathrm{l} / \mathrm{min}$ ), milrinone lactate $(\mathrm{M}, 35 \mu \mathrm{g} /$ $\mathrm{kg} / \mathrm{min}$; i.v. infusion rate, $4 \mu \mathrm{l} / \mathrm{min}$; Sanofi-Synthelabo Inc., New York), or esmolol $\mathrm{HCl}$ (Es, $1 \mathrm{mg} / \mathrm{kg} / \mathrm{min}$; i.v. infusion rate, $4 \mu \mathrm{l} / \mathrm{min}$; Uni Pharma Co., Taiwan) infusion $30 \mathrm{~min}$ after NE infusion (Supplemental Fig. 2). The i.v. infusion rate was determined by the dosage (see "Discussion" section) and the volume according to the practice guideline. ${ }^{22}$

A polyethylene catheter was inserted into the femoral artery for monitoring blood pressure $(\mathrm{BP})$, heart rate $(\mathrm{HR})$, the adequacy of the anesthesia, and the responses to NE and treatment, while electrocardiography was directly detected via platinum electrodes positioned in the subcutaneous tissue of the left and right thorax and left leg, recorded by Gould Amplifiers. At the end of the experiment, the rats were euthanatized by a high dosage of sodium pentobarbital infusion $(150 \mathrm{mg} / \mathrm{kg}$ i.v.). All animal experiments were approved by the Institutional Animal Care and Use Committee and performed under the guidelines for Animal Experiments of Chang Gung Memorial Hospital and Chang Gung University, in accord with the National Institutes of Health guidelines.

\section{Echocardiography}

Echocardiography examination was performed before and at indicated time points after catecholamine infusion till the animal death, which included short- and long-axis M-mode LV chamber size, fractional shortening, and ejection fraction (EF) of LV, using a $12-\mathrm{MHz}$ probe and a Philips Sonos 5500 Ultrasound System (Philips Medical Systems, Amsterdam, Netherlands) by the same two cardiologists.

\section{Mitochondrial extraction and function assay}

The LV homogenate obtained from a Dounce grinder with a loose pestle was centrifuged at $13,500 \times g$ for $10 \mathrm{~min}$, and the pellet was resuspended with ice-cold isolation buffer containing $320 \mathrm{mM}$ sucrose, $1 \mathrm{mM}$ EDTA, 10 $\mathrm{mM}$ Tris- $\mathrm{HCl}, \mathrm{pH}$ 7.4. After centrifugation at $20,000 \times g$ for $15 \mathrm{~min}$ at $4{ }^{\circ} \mathrm{C}$, the mitochondrial layer was extracted by discontinuous Percoll gradient centrifugation according to procedures described previously. ${ }^{23}$ The purity of the mitochondrial-rich fraction was verified by the expression of the mitochondrial prohibitin, ${ }^{23}$ and the concentration of protein extracts was estimated by the method of Bradford with a protein assay kit (Bio-Rad, Hercules, CA).

Activities of individual enzymes were performed immediately after mitochondrial isolation, using a thermostatically regulated ThermoSpectronic spectrophotometer (Thermo Fisher Scientific, Hertfordshire, UK) at least duplicate determination, and expressed as $\mathrm{nmol} / \mu \mathrm{g}$ protein $/ \mathrm{min} .^{23}$ All reagents used in enzyme assays were purchased from Sigma (St. Louis, MO).

For nicotinamide adenine dinucleotide (NADH) cytochrome c reductase (NCCR; complexes I+ III) activity, the mitochondrial fraction $(20 \mu \mathrm{g}$ of protein) was incubated in a mixture containing $50 \mathrm{mM} \mathrm{K} \mathrm{HPO}_{4}$ buffer, pH7.4, $1.5 \mathrm{mM} \mathrm{KCN}, 1 \mathrm{mM} \beta-\mathrm{NADH}, 20 \mu \mathrm{M}$ rotenone at $37^{\circ} \mathrm{C}$ for $2 \mathrm{~min}$. After the addition of $0.1 \mathrm{mM}$ cytochrome $\mathrm{c}$, the reduction of oxidized cytochrome $\mathrm{c}$ was measured as the difference in the presence or absence of rotenone at $550 \mathrm{~nm}$ for $3 \mathrm{~min}$ at $37^{\circ} \mathrm{C}$. The molar extinction coefficient of cytochrome $c$ at $550 \mathrm{~nm}$ was $18,500 \mathrm{M} / \mathrm{cm}$.

Determination of succinate cytochrome $\mathrm{c}$ reductase (SCCR; complexes II + III) activity in the mitochondrial fraction $(30 \mu \mathrm{g})$ was performed in $40 \mathrm{mM}$ $\mathrm{K}_{2} \mathrm{HPO}_{4}$ buffer (pH 7.4), $1.5 \mathrm{mM} \mathrm{KCN}$, supplemented with $20 \mathrm{mM}$ succinate. After a 5-min equilibration at $37^{\circ} \mathrm{C}, 50 \mu \mathrm{M}$ cytochrome $\mathrm{c}$ was added, and the reaction was monitored at $550 \mathrm{~nm}$ for $3 \mathrm{~min}$ at $37^{\circ} \mathrm{C}$.

Cytochrome c oxidase (CCO; complex IV) activity was measured by oxidation of the reduced cytochrome c. The activity was defined as the first-order rate constant and was calculated from the known concentration of ferrocytochrome $c$ and the enzyme amount in the assay mixture. Mitochondrial fraction $(30 \mu \mathrm{g})$ with $500 \mathrm{mM} \mathrm{K} \mathrm{HPO}_{4}$ (pH7.4) was preincubated at $30^{\circ} \mathrm{C}$ for $5 \mathrm{~min}$. A $45 \mu \mathrm{M}$ ferrocytochrome $\mathrm{c}$ was added to start the reaction and monitored at $550 \mathrm{~nm}$ for $3 \mathrm{~min}$ at $30^{\circ} \mathrm{C}$. The background rate was measured after the addition of $1.0 \mu \mathrm{M} \mathrm{K} 3 \mathrm{Fe}(\mathrm{CN}) 6$.

\section{Total protein extraction and western blot}

LVs of sampled hearts were homogenized with a Dounce grinder with a tight pestle in ice-cold lysis buffer and protease inhibitor cocktail (both from Sigma-Aldrich). The homogenate was centrifuged at 13,500 rpm for $30 \mathrm{~min}$, and the supernatant was collected for quantification by the Micro BCA Protein Assay kit (Thermo Fisher Scientific Inc., MA).

Protein samples were subject to $10 \%$ sodium dodecyl sulfatepolyacrylamide gel electrophoresis as described previously, ${ }^{24}$ and transferred onto PVDF transfer membranes (PerkinElmer Life Sciences, Waltham, MA) for $1.5 \mathrm{~h}$ at $4^{\circ} \mathrm{C}$, using a Bio-Rad miniprotein-III wet transfer unit (Bio-Rad). The transfer membranes were then incubated with a blocking solution (5\% nonfat dried milk dissolved in Tris-buffered saline-Tween buffer $(\mathrm{pH} 7.6,10 \mathrm{mM}$ Tris$\mathrm{HCl}, 150 \mathrm{mM} \mathrm{NaCl}$, and $0.1 \%$ Tween 20 ) for $1 \mathrm{~h}$ at room temperature.

The primary antisera used included mouse polyclonal antiserum against Mt complex I, II, III, IV, and V (1:1000; Invitrogen, CA), PGC-1 a (1:1000; Santa Cruz Biotechnology, TX), TFAM (1:2000; Biovision, CA), prohibitin (1:1000; Cell Signaling Technology, MA), GAPDH (1:50,000; Merck Millipore, CA). After wash, membranes were incubated with the secondary antibodies (1:10,000; Jackson ImmunoResearch, West Grove, PA) for $1 \mathrm{~h}$, then with horseradish peroxidase-conjugated goat anti-rabbit IgG or goat antimouse IgG (Jackson ImmunoResearch), and finally detected using an enhanced chemiluminescence Western blot detection system (GE Healthcare Bio-Sciences Corp., Piscataway, NJ). The amount of detected protein was quantified by the ImageJ software $(\mathrm{NIH})$ and was expressed as the ratio to GAPDH or prohibitin protein.

\section{Statistical analysis}

Data are expressed as a mean \pm SEM. The statistical software GraphPad Prism 5.0 (La Jolla, CA) was used for data analysis. The survival rate was analyzed by the Log-rank (Mantel-Cox) test. For hemodynamic experiments or biochemical experiments that involved multiple groups, two-way variance with repeated measures was used to assess group means. This was followed by the Bonferroni test for post hoc assessment of individual means. $P<0.05$ was considered statistically significant.

\section{RESULTS}

\section{Mortality and dynamic hemodynamic alteration}

To evaluate the time-dependent mortality, the hourly survival rates in four experimental conditions were compared. Data of survival rate indicated that the first-hour survival rate after NE 


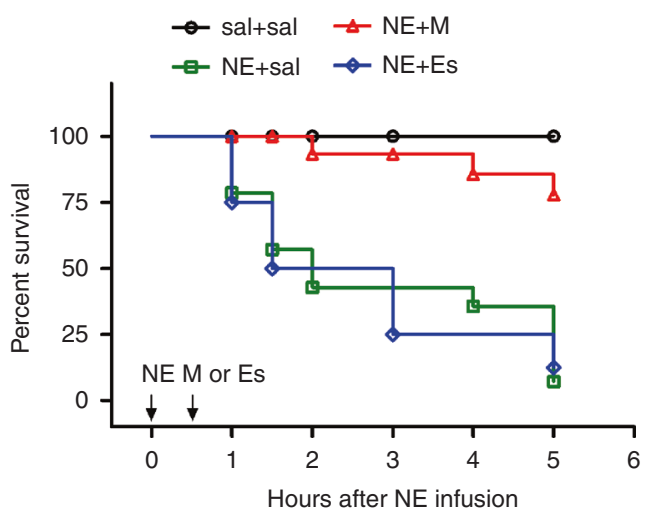

Fig. 1 Decrements of survival rate under intravenous infusion of norepinephrine were reversed by milrinone but not by esmolol. The survival rate was recorded in animals treated with intravenous infusion (i.v.) with saline (sal) or norepinephrine (NE) in combination with sal, milrinone (M), or esmolol (Es) i.v. infusion. The Log-rank (Mantel-Cox) test was used for the survival analyses (sal + sal, $n=$ 20; NE + sal, $n=14 ; \mathrm{NE}+\mathrm{M}, n=20 ; \mathrm{NE}+\mathrm{Es}, n=8$ in the beginning of this experiment).

infusion was around $75 \%$ (Fig. 1). The survival rate was dropped to $50 \%$ at $2 \mathrm{~h}$ after NE infusion. Most animals died around $5 \mathrm{~h}$ after NE infusion. Application with milrinone was able to maintain the survival rate of approximately $80 \%$ after 5 -h NE infusion. Esmolol accelerated the mortality rate to $50 \%$ at $1.5 \mathrm{~h}$. Only $25 \%$ of animals survived after 3-h NE with esmolol infusion. There was no difference in the 5-h survival rate between NE and NE $+E s$ groups. The survival rate in the control group (i.e., saline in combination with saline) was $100 \%$ during the 5 -h observation.

Continuous hypernorepinephrinemia significantly increased HR, and systolic and mean blood pressure (SBP and MBP) (Fig. 2a-C). Esmolol treatment caused a mild depression of NE-induced tachycardia while milrinone treatment did not alter the NEraised tachycardia. Both SBP and MBP were significantly higher in $\mathrm{NE}+$ sal, $\mathrm{NE}+\mathrm{M}$, and $\mathrm{NE}+\mathrm{Es}$ groups in comparison with the control group (Fig. 2b, c). Three hours later, SBP tended to decline in the $N E+M$ and $N E+E s$ groups. Milrinone infusion triggered a smooth decrement of SBP while esmolol infusion induced a sharper decrease of SBP (Fig. 2b).

\section{LV remodeling}

The time-dependent changes of LV sizes were examined by echocardiography. By using M-mode of echocardiography, we found out that the size of LV at diastole (LVIDd) was significantly increased half-an-hour after NE infusion followed by a gradually descending to less than $0.6 \mathrm{~cm}$. Milrinone $(\mathrm{NE}+\mathrm{M})$ induced a further decrease of LVIDd while esmolol treatment (NE + Es) showed no significant alteration when compared with the NE group (Fig. 2d). There was no significant difference in the thickness of the interventricular septum at diastole (IVSd) and EF among groups (Fig. 2e, f).

\section{Mitochondrial oxidative phosphorylation (OXPHOS)}

Mitochondrion is the core of cellular energy production while the increased workload of cardiomyocytes impairs mitochondrial function. We, therefore, investigated the activity of mitochondrial OXPHOS in the heart. Our results indicated that the activities of $\mathrm{NADH}$ cytochrome c reductase (NCCR, Fig. 3a), succinate cytochrome c reductase (SCCR, Fig. 3b), and CCO (Fig. 3c) were significantly suppressed by NE infusion. Milrinone treatment effectively reversed the suppressed activities of NCCR and CCO. On the other hand, esmolol treatment showed no significant effect on impaired OXPHOS.

\section{Mitochondrial biogenesis}

The levels of mtDNA copy numbers and the protein expression of TFAM was significantly suppressed by NE infusion (Fig. 4). Milrinone treatment partially and effectively reversed the suppression of mtDNA copy number and TFAM while esmolol treatment was unable to reverse the suppression.

As the transcription regulator of TFAM and OXPHOS-associated proteins, a decrease of PGC-1a results in the decrease of mitochondrial biogenesis and OXPHOS activity. The protein expression of PGC-1a was significantly suppressed by NE infusion (Fig. 5). Milrinone treatment partially reversed the suppression of PGC-1a while esmolol treatment was unable to reverse the suppression.

\section{DISCUSSION}

In the present study, we delineated the temporal alterations of vital signs and hemodynamics in hypernorepinephrinemiainduced acute $\mathrm{HF}$ and explored the therapeutic effect of milrinone on mitochondrial function and mortality for EV-71-infection mimicry scenario.

Hypernorepinephrinemia causes tachycardia and sequel fulminant HF which occurs in patients with EV-71-brainstem encephalitis, pheochromocytoma, Takotsubo or stress-induced cardiomyopathy, ${ }^{25}$ and possibly in some complications of COVID-19. ${ }^{26,27}$ It is a clinical predicament to judge the progression and the timing of intervention based on the vital signs. Catecholamine may injure the myocardium through an increase in myocardial metabolic demand, ${ }^{28}$ coronary vasospasm, ${ }^{29}$ and a direct toxic effect on calcium overload on cardiac myocytes. ${ }^{30}$ In this study, sustained hypernorepinephrinemia caused a rapid deterioration to animal death within $5 \mathrm{~h}$ and two-phase LV remodeling. HR significantly increased $30 \mathrm{~min}$ since the initial infusion and maintained tachycardia. LV size initially increased and then continued decreasing $1 \mathrm{~h}$ after NE infusion, which revealed that these NE-infused animals rendered LV remodeling in HF with preserved EF status. The temporal alteration of HR during NE infusion seemed to be negatively correlated to LV chamber size. The results from the Fig. 2a, $d$ were partly compatible with the Frank-Starling law-the increases in HR leading to a decrease of preload and consequently a reduced LV size in the $\mathrm{NE}+\mathrm{M}$ group. However, the cardiac output and HF seemed not to get the worst in this group. Of note, this finding may suggest that there is an additional milrinone-triggered cardioprotective effect beyond the traditional hemodynamic physiology. For example, in postconditioning, milrinone can exert its cardioprotection from myocardial infarction via activation of mitochondrial calcium-sensitive potassium channel and release of free radicals. ${ }^{31,32}$ Interestingly, milrinone and esmolol exerted partially opposite effects on such $\mathrm{LV}$ remodeling. The former remained NE-induced tachycardia and reduced LV size more. On the contrary, the latter tended to slightly alleviate NE-induced tachycardia and LV remodeling. Just before animal death, a decrease of HR suddenly occurred from seconds to minutes which was followed by bradycardia, hypotension, and finally a sudden dilatation of LV and depressed EF (echocardiography data not shown). Thus, functional alteration of LV is directly related to mortality and could be a feasible parameter to evaluate the progression of cardiac dysfunction in time.

The hemodynamic evidence indicated that NE infusion dramatically increased SBP. Both milrinone and esmolol gradually attenuated NE-induced hypertension $2.5 \mathrm{~h}$ after treatment. Normalization of SBP seemed not to be a good index of survival. The mechanism of milrinone, a phosphodiesterase inhibitor type 3, works to control acute HF based on its vasodilatation of smooth muscles and increase in cardiac contractility. Thus, it might help maintain coronary circulation against the catecholamine-induced coronary vasospasm in addition to afterload reduction and 

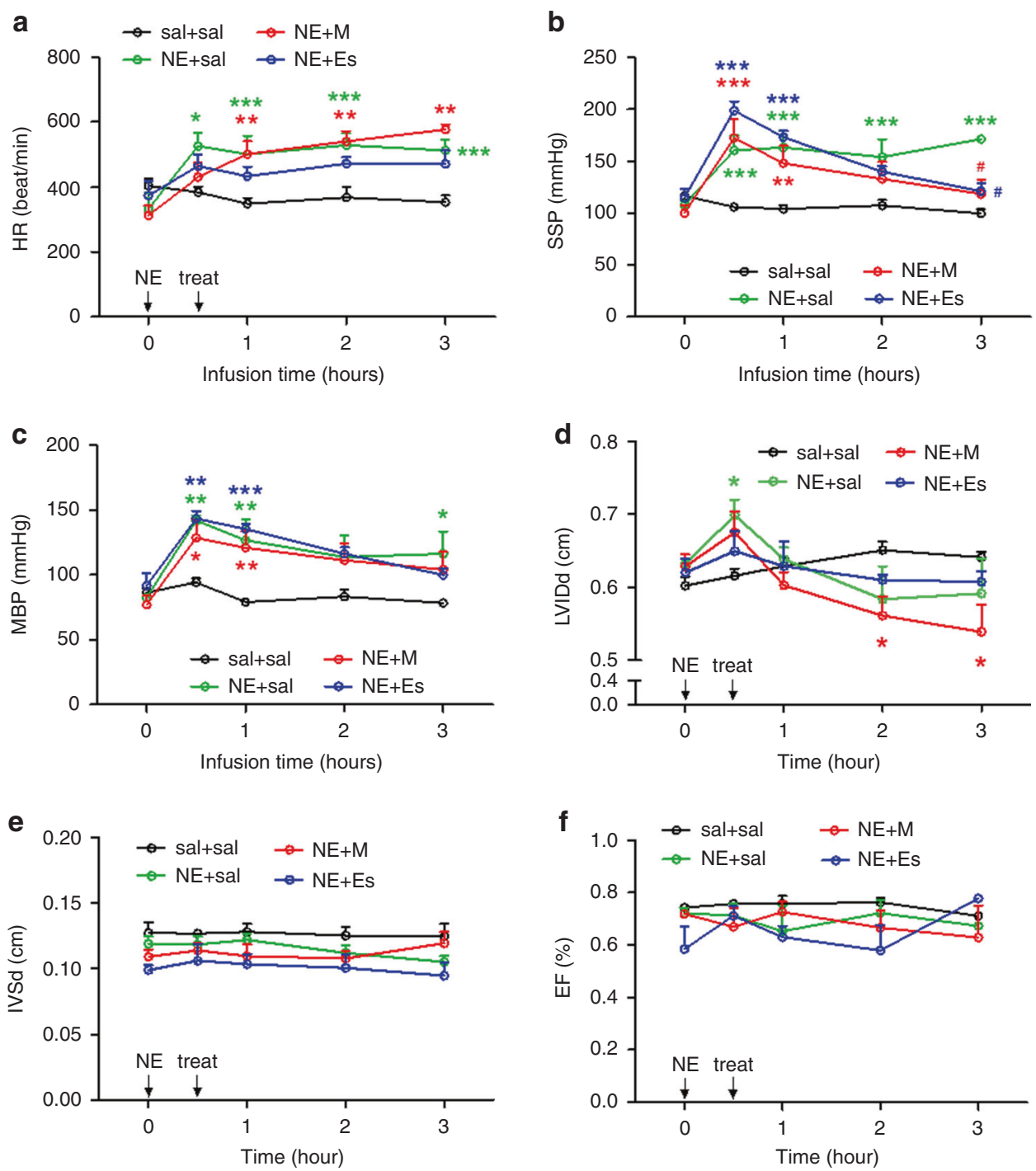

$$
\begin{aligned}
& * * * \mathrm{NE}+\mathrm{M} \text { in comparison with sal+sal, \# in comparison with } \mathrm{NE}+\mathrm{sal} \\
& * * * \mathrm{NE}+\mathrm{sal} \text { in comparison with sal+sal } \\
& * * * \mathrm{NE}+\mathrm{ES} \text { in comparison with sal+sal, \# in comparison with } \mathrm{NE}+\mathrm{sal}
\end{aligned}
$$

Fig. 2 Dynamic alterations of vital signs and echocardiography parameters. a The heart rate (HR), b systolic blood pressure (SBP), and c mean blood pressure (MBP) and $\mathbf{d}$ the size of left ventricle inner diameter at diastole (LVIDd), e the thickness of interventricular septum (IVSd) at diastole, and $\mathbf{f}$ ejection fraction were measured in animals treated with an intravenous infusion with saline (sal) or norepinephrine (NE) in combination with sal, milrinone (M) or esmolol (Es) i.v. infusion. Values are mean \pm SEM $\left(n=6-10\right.$ animals in each experimental group). ${ }^{*} P<$ $0.05,{ }^{* *} P<0.01,{ }^{* * *} P<0.001$ versus sal + sal group, and ${ }^{\#} P<0.05$ versus NE + sal group in two-way ANOVA tests.

inotropic contractility. Esmolol, a selective $\beta-1$ adrenergic antagonist with minor $\beta-2$ blockade which theoretically blocks the NE effect, effectively decreased the SBP, HR, and cardiac oxygen demand but the hemodynamic decrement showed an adverse effect on cardiac function end up with the acceleration of HF. It seems that the $\beta-1$ blocker might result in cardiomyocyte relaxation and decrease cardiac output and could cause hypoxia-associated oxidative stress which deteriorated the progression of HF.

The heart is a high-energy-demanding organ and hypernorepinephrinemia enhances its metabolic demand. ${ }^{33}$ As the result of previous studies, ${ }^{34,35}$ catecholamine could impair mitochondria function in cardiac myocytes. More advanced, we found that NE impaired the mitochondrial activity of ETC, and reduced mitochondrial mass and biogenesis. Milrinone might not only effectively treat the early stage of EV-71-associated fulminant HF as human studies, ${ }^{36}$ but also might partially reverse the mitochondrial OXPHOS and NE-induced mitochondrial dysfunction through increasing mitochondrial biogenesis. Based on these findings, the mitochondrial function may be the determinant factor of survival and cardiac remodeling in such catecholamineinduced $\mathrm{HF}^{37,38}$ The timing of irreversible alteration in mitochondrial dysfunction and cardiac decompensation in such fulminant $\mathrm{HF}$, the critical timing of intervention, and mitochondrial function preservation agents other than milrinone should be investigated in the future.

The limitation of our study was the lack of dose-dependent effects to trigger the fulminant course and to treat for HF. 

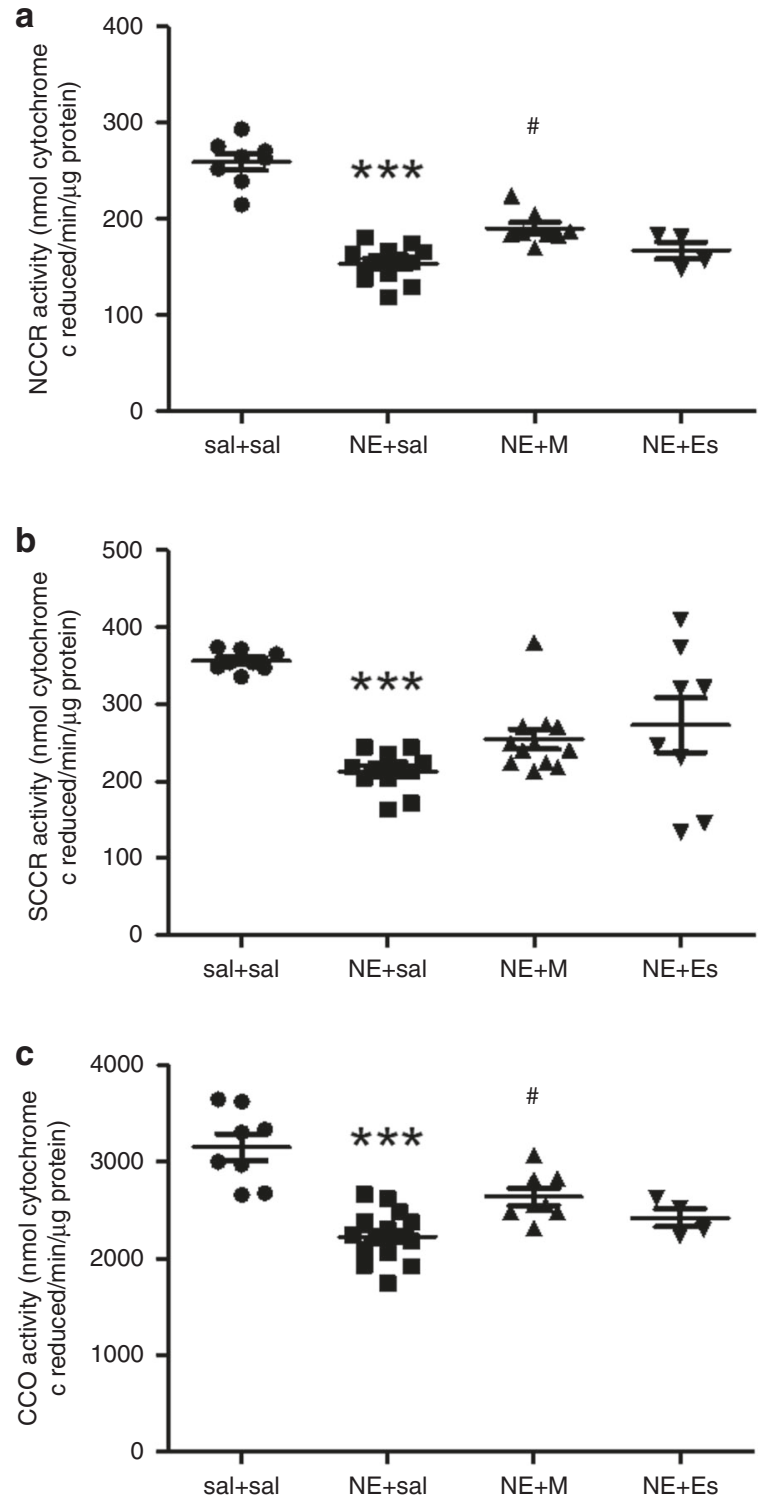

Fig. 3 Mitochondrial respiratory activity of LV myocardium. The levels of mitochondrial respiratory enzyme activities in the LV of the heart, including nicotinamide adenine dinucleotide (NADH) cytochrome $c$ reductase activity $(\mathrm{NCCR}, \mathrm{a})$, succinate cytochrome $\mathrm{c}$ reductase activity (SCCR, b), and cytochrome c oxidase activity (CCO, c), were measured in animals treated with saline (sal) or norepinephrine (NE) in combination with sal, milrinone (M) or esmolol (Es) iv infusion. Values are mean $\pm \operatorname{SEM}(n=6-10$ animals in each experimental group). ${ }^{* * *} P<0.001$ versus sal + sal group, and ${ }^{\#} P<$ 0.05 versus $\mathrm{NE}+$ sal group in the post hoc Tukey's multiple range test.

Compared to other experimental models, ${ }^{34,39}$ our study used a higher dosage of NE to reach the maximal HR approximately up to 1.5-fold of the basal level as clinical manifestations in children of EV-71. ${ }^{1}$ Milrinone $(6.7-8 \mu \mathrm{g} / \mathrm{kg} / \mathrm{min})$ was able to increase the stroke volume and cardiac output. ${ }^{40}$ Esmolol $(0.3 \mathrm{mg} / \mathrm{kg} / \mathrm{min})$ might improve the cardiac function in the sepsis animal model. ${ }^{41}$ We initially treated NE-infused animals with milrinone and esmolol, at the doses of $10 \mu \mathrm{g} / \mathrm{kg} / \mathrm{min}$ and $0.5 \mathrm{mg} / \mathrm{kg} / \mathrm{min}$, respectively. Neither the primary outcome-animal death-nor the secondary outcome-anti-hypertensive effect-was achieved. Therefore, higher doses of milrinone and esmolol were used to optimally lower either HR or SBP.

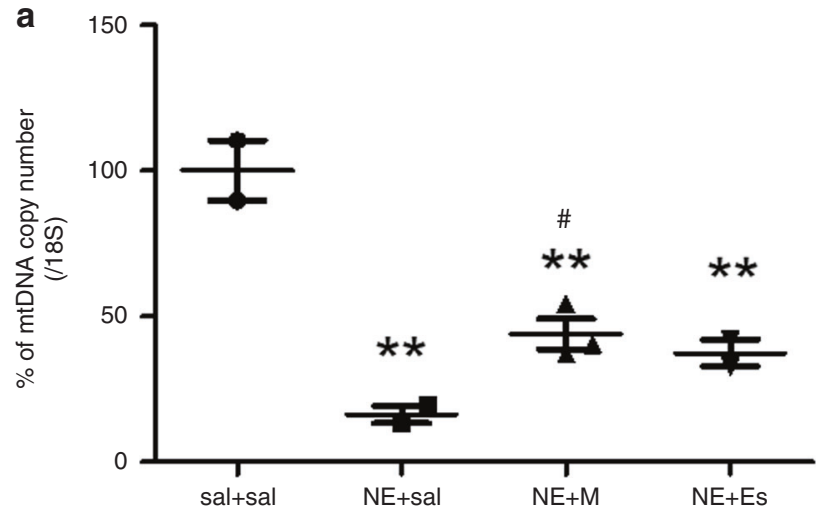

b
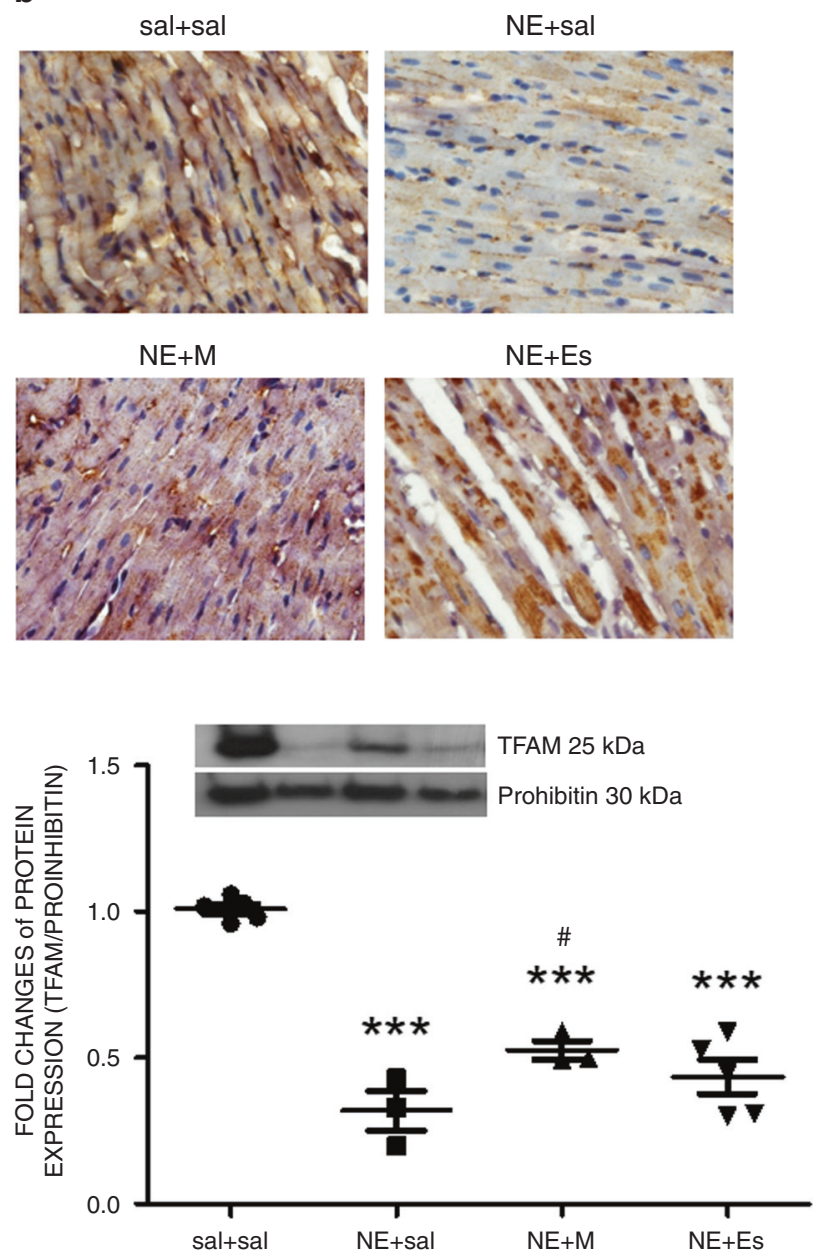

Fig. 4 Mitochondrial DNA (mtDNA) copy numbers and transcription factor A (TFAM) expression levels of LV myocardium. a The copy numbers of mtDNA; b representative immunohistochemical images and gels (inset), and densitometric analysis from western blot showed the expression of TFAM in LV of heart from animals treated with saline (sal) or norepinephrine (NE) in combination with sal, milrinone (M), or esmolol (Es). Values are mean \pm SEM $(n=8-10$ animals in each experimental group). ${ }^{* *} P<0.01,{ }^{* *} P<0.001$ versus sal + sal group, and ${ }^{\#} P<0.05$ versus $N E+$ sal group in the post hoc Tukey's multiple range test.

\section{CONCLUSIONS}

In the present study, sustained hypernorepinephrinemia provoked two-phase LV remodeling and high mortality. Maintenance of 
a

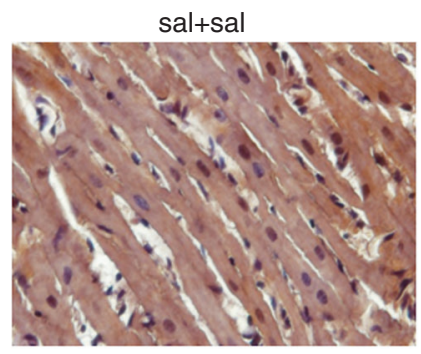

$\mathrm{NE}+\mathrm{M}$

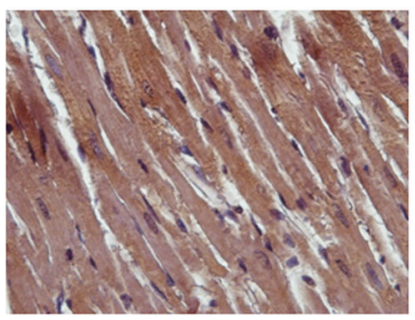

b

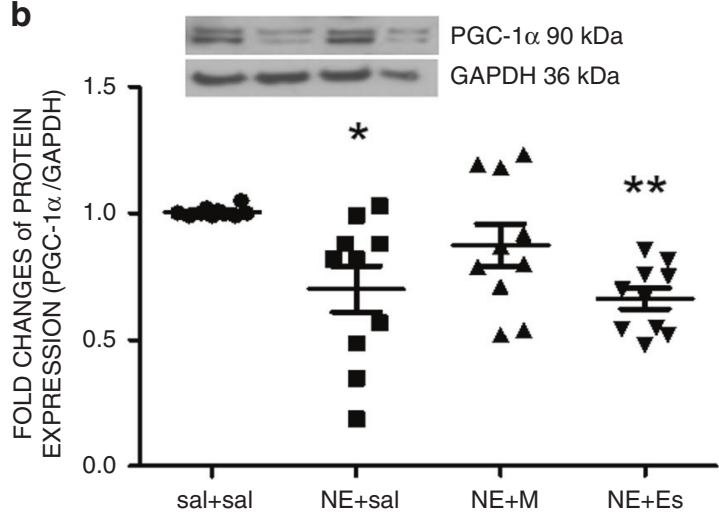

Fig. 5 The expression of peroxisome proliferator-activated receptor-gamma coactivator 1-alpha (PGC-1a) of LV myocardium. a Representative immunohistochemical images; b representative gels (inset) and densitometric analysis from western blot showed the expression of PGC-1 $\alpha$ in the LV of heart from animals treated with saline (sal) or norepinephrine (NE) in combination with sal, milrinone (M), or esmolol (Es). Values are mean \pm SEM $(n=8-10$ animals in each experimental group). ${ }^{*} P<0.05,{ }^{*} P<0.01$ versus sal + sal group in the post hoc Tukey's multiple range test.

mitochondrial function may be a potential therapeutic strategy in such catecholamine-induced HF.

\section{REFERENCES}

1. Fu, Y. C. et al. Cardiac complications of enterovirus rhombencephalitis. Arch. Dis. Child. 89, 368-373 (2004).

2. Spapen, J., de Filette, J., Lochy, S. \& Spapen, H. Acute heart failure as a first presentation of pheochromocytoma complicated with "inverted" Takotsubo syndrome. Case Rep. Endocrinol. 2020, 2521046 (2020).

3. Park, J. H. et al. Prevalence and patterns of left ventricular dysfunction in patients with pheochromocytoma. J. Cardiovasc. Ultrasound 19, 76-82 (2011).

4. Liu, P. P., Blet, A., Smyth, D. \& Li, H. The science underlying Covid-19: implications for the cardiovascular system. Circulation 142, 68-78 (2020).

5. Petramala, L. et al. Cardiomyopathies and adrenal diseases. Int. J. Mol. Sci. 21, 5047 (2020).

6. Ucakturk, S. A. et al. Catecholamine-induced myocarditis in a child with pheochromocytoma. J. Clin. Res. Pediatr. Endocrinol. 12, 202-205 (2020).

7. Liao, Y. T., Wang, S. M., Wang, J. R., Yu, C. K. \& Liu, C. C. Norepinephrine and epinephrine enhanced the infectivity of enterovirus 71. PLOS ONE 10, e0135154 (2015).
8. Kochi, A. N., Tagliari, A. P., Forleo, G. B., Fassini, G. M. \& Tondo, C. Cardiac and arrhythmic complications in patients with Covid-19. J. Cardiovasc. Electrophysiol. 31, 1003-1008 (2020).

9. Fu, Y. C. et al. Norepinephrine induces apoptosis in neonatal rat cardiomyocytes through a reactive oxygen species-TNF alpha-caspase signaling pathway. Cardiovasc. Res. 62, 558-567 (2004).

10. Fu, Y. C., Yin, S. C., Chi, C. S., Hwang, B. \& Hsu, S. L. Norepinephrine induces apoptosis in neonatal rat endothelial cells via a Ros-dependent Jnk activation pathway. Apoptosis 11, 2053-2063 (2006).

11. LV, X. X. et al. Berberine inhibits norepinephrine-induced apoptosis in neonatal rat cardiomyocytes via inhibiting Ros-Tnf-alpha-caspase signaling pathway. Chin. J. Integr. Med. 19, 424-431 (2013).

12. Feldman, D. S., Carnes, C. A., Abraham, W. T. \& Bristow, M. R. Mechanisms of disease: beta-adrenergic receptors-alterations in signal transduction and pharmacogenomics in heart failure. Nat. Clin. Pract. Cardiovasc. Med. 2, 475-483 (2005).

13. Izem-Meziane, M. et al. Catecholamine-induced cardiac mitochondrial dysfunction and MPTP opening: protective effect of curcumin. Am. J. Physiol. Heart Circ. Physiol. 302, H665-H674 (2012).

14. Liaudet, L., Calderari, B. \& Pacher, P. Pathophysiological mechanisms of catecholamine and cocaine-mediated cardiotoxicity. Heart Fail. Rev. 19, 815-824 (2014).

15. Wu, F., Zhang, J. \& Beard, D. A. Experimentally observed phenomena on cardiac energetics in heart failure emerge from simulations of cardiac metabolism. Proc. Natl Acad. Sci. USA 106, 7143-7148 (2009).

16. Lemieux, H., Semsroth, S., Antretter, H., Hofer, D. \& Gnaiger, E. Mitochondrial respiratory control and early defects of oxidative phosphorylation in the failing human heart. Int. J. Biochem. Cell Biol. 43, 1729-1738 (2011).

17. Rosca, M., Minkler, P. \& Hoppel, C. L. Cardiac mitochondria in heart failure: normal cardiolipin profile and increased threonine phosphorylation of complex IV. Biochim. Biophys. Acta 1807, 1373-1382 (2011).

18. Mortensen, S. A. et al. The effect of coenzyme Q10 on morbidity and mortality in chronic heart failure: results from Q-Symbio: a randomized double-blind trial. JACC Heart Fail. 2, 641-649 (2014).

19. Daubert, M. A. et al. Novel mitochondria-targeting peptide in heart failure treatment: a randomized, placebo-controlled trial of elamipretide. Circ. Heart Fail. 10, e004389 (2017).

20. Pierce, J. D. et al. Study protocol, randomized controlled trial: reducing symptom burden in patients with heart failure with preserved ejection fraction using ubiquinol and/or D-ribose. BMC Cardiovasc. Disord. 18, 57 (2018).

21. Rimbaud, S., Garnier, A. \& Ventura-Clapier, R. Mitochondrial biogenesis in cardiac pathophysiology. Pharmacol. Rep. 61, 131-138 (2009).

22. Morton, D. B. et al. Refining procedures for the administration of substances. Report of the BVAAWF/FRAME/RSPCA/UFAW Joint Working Group on Refinement. British Veterinary Association Animal Welfare Foundation/Fund for the Replacement of Animals in Medical Experiments/Royal Society for the Prevention of Cruelty to Animals/Universities Federation for Animal Welfare. Lab. Anim. 35, 1-41 (2001).

23. Wu, K. L., Hsu, C. \& Chan, J. Y. Impairment of the mitochondrial respiratory enzyme activity triggers sequential activation of apoptosis-inducing factordependent and caspase-dependent signaling pathways to induce apoptosis after spinal cord injury. J. Neurochem. 101, 1552-1566 (2007).

24. Wu, K. L., Chan, S. H. \& Chan, J. Y. Neuroinflammation and oxidative stress in rostral ventrolateral medulla contribute to neurogenic hypertension induced by systemic inflammation. J. Neuroinflammation 9, 212 (2012).

25. Lyon, A. R., Rees, P. S., Prasad, S., Poole-Wilson, P. A. \& Harding, S. E. Stress (Takotsubo) cardiomyopathy-a novel pathophysiological hypothesis to explain catecholamine-induced acute myocardial stunning. Nat. Clin. Pract. Cardiovasc. Med. 5, 22-29 (2008).

26. Giustino, G. et al. Takotsubo cardiomyopathy in Covid-19. J. Am. Coll. Cardiol. 76, 628-629 (2020).

27. Kariyanna, P. T. et al. Apical Takotsubo cardiomyopathy in a Covid-19 patient presenting with stroke: a case report and pathophysiologic insights. Am. J. Med. Case Rep. 8, 350-357 (2020).

28. Wang, T. D., Wu, C. C. \& Lee, Y. T. Myocardial stunning after cerebral infarction. Int. J. Cardiol. 58, 308-311 (1997).

29. Oppenheimer, S. M., Gelb, A., Girvin, J. P. \& Hachinski, V. C. Cardiovascular effects of human insular cortex stimulation. Neurology 42, 1727-1732 (1992).

30. Nguyen, H. \& Zaroff, J. G. Neurogenic stunned myocardium. Curr. Neurol. Neurosci. Rep. 9, 486-491 (2009).

31. Behmenburg, F. et al. Milrinone-induced postconditioning requires activation of mitochondrial $\mathrm{Ca}(2+)$-sensitive potassium $\left(\mathrm{mBK}_{\mathrm{Ca}}\right)$ channels. J. Cardiothorac. Vasc. Anesth. 32, 2142-2148 (2018).

32. Raupach, A. et al. Milrinone-induced pharmacological preconditioning in cardioprotection: hints for a role of mitochondrial mechanisms. J. Clin. Med. 8, 507 (2019).

33. Huss, J. M. \& Kelly, D. P. Mitochondrial energy metabolism in heart failure: a question of balance. J. Clin. Investig. 115, 547-555 (2005). 
34. Sobel, B., Jequier, E., Sjoerdsma, A. \& Lovenberg, W. Effect of catecholamines and adrenergic blocking agents on oxidative phosphorylation in rat heart mitochondria. Circ. Res. 19, 1050-1061 (1966).

35. Willis, B. C. et al. Impaired oxidative metabolism and calcium mishandling underlie cardiac dysfunction in a rat model of post-acute isoproterenol-induced cardiomyopathy. Am. J. Physiol. Heart Circ. Physiol. 308, H467-H477 (2015).

36. Chi, C. Y. et al. Milrinone therapy for enterovirus 71-induced pulmonary edema and/or neurogenic shock in children: a randomized controlled trial. Crit. Care Med. 41, 1754-1760 (2013)

37. Sabbah, H. N. Targeting the mitochondria in heart failure: a translational perspective. JACC Basic Transl. Sci. 5, 88-106 (2020).

38. Brown, D. A. et al. Expert Consensus Document: mitochondrial function as a therapeutic target in heart failure. Nat. Rev. Cardiol. 14, 238-250 (2017)

39. Irlbeck, M., Muhling, O., Iwai, T. \& Zimmer, H. G. Different response of the rat left and right heart to norepinephrine. Cardiovasc. Res. 31, 157-162 (1996).

40. Tveita, T. \& Sieck, G. C. Effects of milrinone on left ventricular cardiac function during cooling in an intact animal model. Cryobiology 65, 27-32 (2012).

41. Wei, C. et al. Effects of low doses of esmolol on cardiac and vascular function in experimental septic shock. Crit. Care 20, 407 (2016).

\section{AUTHOR CONTRIBUTIONS}

I.-C.L.: conception and design of the study, acquisition, analysis, and interpretation of data, and drafting and revising the manuscript. K.L.H.W.: conception and design of the study, analysis, and interpretation of data, and drafting and revising the manuscript. C.-W.W., Y.-J.L., and M.-H.L.: acquisition, analysis, and interpretation of data, and revising the manuscript. K.-S.H. and J.Y.H.C.: supervision of data acquisition and revising the manuscript. All authors made final approval of the version and agreed to be accountable for all aspects of the work.

\section{FUNDING}

This work was supported by grant CMRPG8G0571 from Chang Gung Memorial Hospital, Kaohsiung, Taiwan to I.-C.L. and M.-H.L.

\section{COMPETING INTERESTS}

The authors declare no competing interests.

\section{ETHICS APPROVAL AND CONSENT TO PARTICIPATE}

Patient consent was not required.

\section{ADDITIONAL INFORMATION}

Supplementary information The online version contains supplementary material available at https://doi.org/10.1038/s41390-022-01964-6.

Correspondence and requests for materials should be addressed to I-Chun Lin or Kay L. H. Wu.

Reprints and permission information is available at http://www.nature.com/ reprints

Publisher's note Springer Nature remains neutral with regard to jurisdictional claims in published maps and institutional affiliations. 